\title{
Noninteracting Control with Stability for Hamiltonian Systems
}

\author{
Alessandro Astolfi and Laura Menini
}

\begin{abstract}
The problem of noninteraction with stability via dynamic state feedback is addressed and solved for a class of nonlinear Hamiltonian systems. A simple way to check necessary and sufficient condition is proposed. It is well known that to decide if the problem is solvable, and which class of state feedback has to be used, the stability properties of some special dynamics are to be investigated. For this reason, on the way to the main result, it is shown that such dynamics are not necessarily Hamiltonian. Several examples, clarifying the role of different classes of state-feedback control laws (either static or dynamic) in the solution of the problem, are proposed.
\end{abstract}

Index Terms-Geometric control, Hamiltonian systems, noninteracting control, nonlinear control.

\section{INTRODUCTION AND MOTIVATIONS}

$\mathbf{T}$ HE PROBLEM of noninteraction with stability for nonlinear systems has been studied by several authors; see [4], [9], [7], [11], [14], and [6], as well as the monograph [2]. Necessary and sufficient conditions for the existence of either static or dynamic state-feedback control laws, which allow one to obtain stable noninteractive closed-loop control systems, have been proposed, and systematic procedures for the design of such control laws have been given. Despite the elegant characterization of the problem, which is based on geometric control theory, the applicability of the theory to physical systems has not received (to the best of the authors knowledge) enough interest. A notable exception is [5]. Therein, the problem of noninteraction with stability is addressed for the class of (nonlinear) Hamiltonian systems by means of a particular class of state-feedback control laws. It has been shown in [5] that Hamiltonian systems can be put in a particular canonical form, which will be exploited in this paper too, since it highly facilitates the analysis of the problem. As a matter of fact, by using such a canonical form, it is easier to take into account the well-known fact, proven in [10, Ch. 12], that the zero dynamics of Hamiltonian systems are Hamiltonian. In [5] it is shown that, if a particular class of static state-feedback control laws is considered, noninteraction and asymptotic stability are not jointly achievable for Hamiltonian systems whose zero dynamics are nontrivial. Nevertheless, under suitable hypotheses, noninteraction with simple stability can be obtained,

Manuscript received March 19, 1998; revised April 10, 1999. Recommended by Associate Editors, A. Bloch and P. Crouch.

A. Astolfi is with Imperial College, London SW7 2BT, U.K. and also with the Politecnico di Milano, Milan 20133, Italy (e-mail: a.astolfi@ic.ac.uk).

L. Menini is with the Università di Roma Tor Vergata, Rome 00133, Italy (e-mail: menini@disp.uniroma2.it).

Publisher Item Identifier S 0018-9286(00)06312-1. as shown in [5], by means of static state-feedback control laws in the mentioned class.

The work reported in this paper stems from the consideration that, by using more general state-feedback control laws, either static or dynamic, the problem of noninteracting control with stability can be solved for larger classes of systems. It is well known (see [13], [8], and [12]) that, in the case of linear systems, the problem can be solved by means of dynamic state-feedback for all those systems for which the two problems of stabilization and of noninteraction are separately solvable. This implies that, if dynamic state-feedback is allowed, the problem of noninteraction with asymptotic stability is generically solvable for linear controllable Hamiltonian systems.

The importance of Hamiltonian systems in the modeling of practical situations is well known (see [10, Ch. 12] and the concise exposition in [1]), hence it seems of interest to investigate if the general results concerning the problem of noninteracting control with stability, reported in [2] and [6], assume special characterization when applied to general, nonlinear, Hamiltonian systems, in view of their special properties.

The main result of this paper, reported in Section IV, consists of a simple to check condition for the solvability of the problem of noninteraction with stability, which is necessary and sufficient for the class of systems considered and can be used with respect to different stability requirements. In what follows, all the proposed results are local, i.e., valid in a suitable neighborhood of the equilibrium configuration of the system.

On the way to the main result, it is also shown that, contrarily to what holds true for the zero dynamics, the $P^{\perp}, P^{*}$, and $\Delta_{\text {mix }}$ dynamics ${ }^{1}$ of general Hamiltonian systems are not necessarily Hamiltonian. Such facts are shown, in Section III, by means of simple low-order examples. In particular, since all these dynamics are contained in the zero dynamics, they may not be Hamiltonian if their dimension is smaller than that of the zero dynamics. Since the mentioned dynamics are responsible for obstructions to the solvability of the problem (if either the $P^{\perp}$ or the $\Delta_{\text {mix }}$ dynamics is not "stable," then the problem is not solvable, whereas if the $P^{*}$ dynamics are not "stable," then the problem is not solvable by means of static state feedback, but may be solvable by means of dynamic state feedback), it follows that their "stability" has to be checked in order to decide 1) if the problem is indeed solvable and 2) in case it is, which class of control laws can be adopted.

Finally, in Section VI, two mechanical systems are studied. In the first one, the problem of noninteraction with (simple) stability is solved by means of dynamic state feedback for a system

${ }^{1}$ Definitions are reported in Section III. 
having an unstable zero dynamics; in the second one, it is shown that the problem of noninteraction with asymptotic stability in the first approximation can be solved by means of static state feedback, even if the zero dynamics are nontrivial.

As a result of the considerations carried out in this paper, the class of Hamiltonian systems for which the problem of noninteracting control with stability can be solved is enlarged with respect to the results available in the existing literature.

In what follows, $e_{i}$ denotes the $i$ th column of the identity matrix of proper dimension, $\emptyset$ denotes the zero distribution, and $\{\emptyset\}$ denotes the empty set.

\section{BACKGROUND ON HAMILTONIAN SYSTEMS}

In this paper, the problem of noninteracting control with stability will be tackled for a class of two inputs-two outputs Hamiltonian systems, namely, systems described, in Hamiltonian form, by means of the following equations:

$$
\begin{aligned}
& \dot{q}_{i}=\frac{\partial H(q, p, u)}{\partial p_{i}}, \quad i=1, \cdots, n \\
& \dot{p}_{i}=-\frac{\partial H(q, p, u)}{\partial q_{i}}, \quad i=1, \cdots, n \\
& y_{1}=q_{1}, \quad y_{2}=q_{2}
\end{aligned}
$$

where the Hamiltonian function $H(q, p, u)$ has the form

$$
H(q, p, u)=H_{0}(q, p)-q_{1} u_{1}-q_{2} u_{2}
$$

and is assumed to be sufficiently smooth.

The components $q_{i}, i=1,2, \cdots, n, n \geq 2$, of the vector $q$ are suitable configuration coordinates, whereas the components $p_{i}, i=1,2, \cdots, n$, of the vector $p$ are the corresponding generalized momenta; the first two degrees of freedom $q_{1}$ and $q_{2}$ are actuated by means of the external inputs $u_{1}, u_{2}$. Note that $y_{1}$ and $y_{2}$ are the "natural outputs" (see [10, Ch. 12]). A subclass of Hamiltonian systems of special importance is that of simple Hamiltonian systems, in which the function $H_{0}$ has the form

$$
H_{0}(q, p)=\frac{1}{2} p^{T} G(q) p+V(q) .
$$

The square $n$-dimensional symmetric matrix $G(q)$ is assumed to be positive definite for every $q$ in its domain: this condition is satisfied by many Hamiltonian systems of practical interest, e.g., by those representing mechanical systems, in which the term $(1 / 2) p^{T} G(q) p$ corresponds to the kinetic energy. Moreover, it is also assumed that $(\partial V / \partial q)(0)=0$, so that the point $q=0$, $p=0$ is an equilibrium point for system (1).

Let $x:=\left[\begin{array}{ll}q^{T} & p^{T}\end{array}\right]^{T}$, and let the vector fields $f(x), g_{1}, g_{2}$, $h_{1}(x), h_{2}(x)$, be given by

$$
\begin{aligned}
f_{i}(x) & :=\frac{\partial H_{0}(q, p)}{\partial p_{i}}, \quad i=1,2, \cdots, n \\
f_{n+i}(x) & :=-\frac{\partial H_{0}(q, p)}{\partial q_{i}}, \quad i=1,2, \cdots, n \\
g_{1} & =e_{n+1}, \quad g_{2}=e_{n+2} \\
h_{1}(x) & :=q_{1}, \quad h_{2}(x):=q_{2}
\end{aligned}
$$

so that (1) can be rewritten as

$$
\begin{aligned}
\dot{x} & =f(x)+g_{1} u_{1}+g_{2} u_{2} \\
y_{1} & =h_{1}(x), \quad y_{2}=h_{2}(x) .
\end{aligned}
$$

Let the characteristic numbers $\rho_{1}, \rho_{2}$, of system (1) at the point $q=0, p=0$ be defined as in [10, Definition 8.7], i.e.,

$$
\begin{gathered}
\rho_{i}=\min _{k \in \mathbb{Z}^{+}}\left\{L_{g_{j}} L_{f}^{l} h_{i}(x)=0, \forall l=0,1, \cdots, k-1,\right. \\
\forall j=1,2, \forall x \text { in a neighborhood of } 0, \\
\left.\exists j: L_{g_{j}} L_{f}^{k} h_{i}(0) \neq 0\right\}, \quad i=1,2
\end{gathered}
$$

and let the decoupling matrix $A(\cdot)$ be defined, in a neighborhood of the origin, as the two-dimensional square matrix

$$
A(x):=\left[\begin{array}{cc}
L_{g_{1}} L_{f}^{\rho_{1}} h_{1}(x) & L_{g_{2}} L_{f}^{\rho_{1}} h_{1}(x) \\
L_{g_{1}} L_{f}^{\rho_{2}} h_{2}(x) & L_{g_{2}} L_{f}^{\rho_{2}} h_{2}(x)
\end{array}\right] .
$$

In the case of simple Hamiltonian systems, it is well known [10, Ch. 12] that $\rho_{1}=\rho_{2}=1$ and that $A(x)=G_{11}(q)$, where $G_{11}(q)$ is the two $\times$ two leading submatrix of $G(q)$. Therefore, the matrix $A(x)$ is nonsingular everywhere. Consider a feedback control law of the form

$$
u=\alpha(x)+\beta(x) v
$$

where $\alpha(x)$ and $\beta(x)$ are defined as

$$
\begin{aligned}
\alpha(x) & :=-(A(x))^{-1} b(x), \quad \beta(x):=(A(x))^{-1} \\
b(x) & :=\left[\begin{array}{l}
L_{f}^{2} h_{1}(x) \\
L_{f}^{2} h_{2}(x)
\end{array}\right]
\end{aligned}
$$

and $v=\left[v_{1} v_{2}\right]^{T}$ is the vector of the new inputs. If the vectors $\xi_{i}$ are given by

$$
\begin{aligned}
& \xi_{1}=\left[\begin{array}{c}
h_{1}(x) \\
L_{f} h_{1}(x)
\end{array}\right]=\left[\begin{array}{c}
q_{1} \\
\dot{q}_{1}
\end{array}\right] \\
& \xi_{2}=\left[\begin{array}{c}
h_{2}(x) \\
L_{f} h_{2}(x)
\end{array}\right]=\left[\begin{array}{c}
q_{2} \\
\dot{q}_{2}
\end{array}\right]
\end{aligned}
$$

and the vectors $\tilde{q}$ and $\tilde{p}$ are given by $\tilde{q}=\left[q_{3}, \cdots, q_{n}\right]^{T}, \tilde{p}=$ $\left[p_{3}, \cdots, p_{n}\right]^{T}$, the closed-loop system can be written as

$$
\begin{aligned}
\dot{\xi}_{1} & =A \xi_{1}+B v_{1} \\
\dot{\xi}_{2} & =A \xi_{2}+B v_{2} \\
\dot{\tilde{q}} & =\frac{\partial \hat{H}}{\partial \tilde{p}}\left(\tilde{q}, \tilde{p}, \xi_{1}, \xi_{2}\right)+R_{1}\left(\tilde{q}, \tilde{p}, \xi_{1}, \xi_{2}\right) \\
\dot{\tilde{p}} & =-\frac{\partial \hat{H}}{\partial \tilde{q}}\left(\tilde{q}, \tilde{p}, \xi_{1}, \xi_{2}\right)+R_{2}\left(\tilde{q}, \tilde{p}, \xi_{1}, \xi_{2}\right) \\
y_{1} & =[10] \xi_{1} \\
y_{2} & =[10] \xi_{2}
\end{aligned}
$$

where

$$
A=\left[\begin{array}{ll}
0 & 1 \\
0 & 0
\end{array}\right] \quad B=\left[\begin{array}{l}
0 \\
1
\end{array}\right]
$$

$\hat{H}\left(\tilde{q}, \tilde{p}, \xi_{1}, \xi_{2}\right)$ is the function $H_{0}(q, p)$, written in the new coordinates, and $R_{1}\left(\tilde{q}, \tilde{p}, \xi_{1}, \xi_{2}\right), R_{2}\left(\tilde{q}, \tilde{p}, \xi_{1}, \xi_{2}\right)$ are suitable functions such that $R_{i}(\tilde{q}, \tilde{p}, 0,0)=0, i=1,2$, for all $\tilde{q}, \tilde{p}$ in a neighborhood of the origin.

For simple Hamiltonian systems, the results in [5], [10, Ch. 12], and [6, Ch. 6] imply that if $\Delta^{*}$ denotes the largest locally controlled invariant distribution contained in $\operatorname{Ker}\left(d h_{1}\right) \cap$ $\operatorname{Ker}\left(d h_{2}\right)$, then

$$
\Delta^{*}=\operatorname{span}\left\{\frac{\partial}{\partial \tilde{q}_{1}}, \cdots, \frac{\partial}{\partial \tilde{q}_{n-2}}, \frac{\partial}{\partial \tilde{p}_{1}}, \cdots, \frac{\partial}{\partial \tilde{p}_{n-2}}\right\}
$$


hence $\tilde{q}$ and $\tilde{p}$ can be used to describe the zero dynamics of the system (1), (3), i.e.,

$$
\begin{aligned}
\dot{\tilde{q}} & =\frac{\partial \tilde{H}}{\partial \tilde{p}}(\tilde{q}, \tilde{p}) \\
\dot{\tilde{p}} & =-\frac{\partial \tilde{H}}{\partial \tilde{q}}(\tilde{q}, \tilde{p})
\end{aligned}
$$

where the restricted Hamiltonian (see [10, Ch. 12]) $\tilde{H}(\tilde{q}, \tilde{p}):=$ $\hat{H}(\tilde{q}, \tilde{p}, 0,0)$ can be computed explicitly by means of the following equation:

$\tilde{H}(\tilde{q}, \tilde{p})=\frac{1}{2} \tilde{p}^{T}\left(G_{22}-G_{12}^{T} G_{11}^{-1} G_{12}\right)\left(\left[\begin{array}{l}0 \\ \tilde{q}\end{array}\right]\right) \tilde{p}+V\left(\left[\begin{array}{l}0 \\ \tilde{q}\end{array}\right]\right)$.

Note that (10) coincides with (9c) and (9d) for $\xi_{1}=\xi_{2}=0$.

As observed in [5], the zero dynamics of general Hamiltonian systems of the form (1) are Hamiltonian; for simple Hamiltonian systems, this implies that if $n>2$, any "decentralized" feedback control law described by the equations

$$
\begin{aligned}
& v_{1}=\epsilon_{11} q_{1}+\epsilon_{12} \dot{q}_{1} \\
& v_{2}=\epsilon_{21} q_{2}+\epsilon_{22} \dot{q}_{2}
\end{aligned}
$$

with $\epsilon_{i j} \in \mathbb{R}$, cannot achieve asymptotic stability for the closed-loop system. Nevertheless, if $(0,0)$ is a stable equilibrium point for (10), (simple) stability can be achieved by a proper choice of the gains $\epsilon_{11}, \epsilon_{12}, \epsilon_{21}, \epsilon_{22}$, as discussed in [5].

In this paper, it is shown that, allowing more general statefeedback control laws, static or dynamic, the problem of noninteracting control with stability (simple or asymptotic, depending on the properties of the given system) can be solved for a wider class of Hamiltonian systems.

\section{SOME GeOMETRIC PROPERTIES}

Given a general nonlinear system of the form

$$
\begin{aligned}
& \dot{\bar{x}}=\bar{f}(\bar{x})+\bar{g}(\bar{x}) \bar{u} \\
& \bar{y}=\bar{h}(\bar{x})
\end{aligned}
$$

with $\bar{x} \in \mathbb{R}^{n}, \bar{u}, \bar{y} \in \mathbb{R}^{m}, \bar{f}(0)=0$ and $\bar{h}(0)=0$ satisfying suitable regularity assumptions (see [2] and [6, Ch. 7]), several approaches can be adopted in order to solve the problem of noninteraction with stability, depending on the geometric properties of (12). The main results of the general theory will be now summarized; to this purpose, some notations and well-known properties are recalled. For the sake of simplicity, in the first half of the present section, the exposition will be limited to the case in which the stability requirement is that of asymptotic stability in the first approximation. For the objectives to be pursued in this paper, it is sufficient to restrict the attention to the class of systems for which the characteristic numbers $\rho_{i}, i=1,2, \cdots, m$, can be defined, similarly to (5), and the decoupling matrix $\bar{A}(\bar{x})$, also defined similarly to what has been done for two input-two output systems, is nonsingular at $\bar{x}=0$. Consider any regular static state-feedback control law $\bar{u}$, of the form

$$
\bar{u}=\bar{\alpha}(\bar{x})+\bar{\beta}(\bar{x}) \bar{v}
$$

such that the closed-loop system is noninteractive [such a control law exists by virtue of the assumptions on matrix $\bar{A}(\bar{x})]$ and rewrite the closed-loop system (12), (13) as follows:

$$
\begin{aligned}
& \dot{\bar{x}}=\tilde{f}(\bar{x})+\tilde{g}(\bar{x}) \bar{v} \\
& \bar{y}=\bar{h}(\bar{x})
\end{aligned}
$$

i.e., define $\tilde{f}(\bar{x}):=\bar{f}(\bar{x})+\bar{g}(\bar{x}) \bar{\alpha}(\bar{x})$ and $\tilde{g}(\bar{x}):=\bar{g}(\bar{x}) \bar{\beta}(\bar{x})$. Let the distributions $P$ and $P^{*}$ be defined as follows:

$$
\begin{aligned}
P & =\left\langle\bar{f}, \bar{g}_{1}, \bar{g}_{2}, \cdots, \bar{g}_{m} \mid \operatorname{span}\left\{\bar{g}_{j}, j=1,2, \cdots, m\right\}\right\rangle \\
P^{*} & =\bigcap_{i=1}^{m} P_{i}^{*}
\end{aligned}
$$

where

$$
P_{i}^{*}=\left\langle\tilde{f}, \tilde{g}_{1}, \tilde{g}_{2}, \cdots, \tilde{g}_{m} \mid \operatorname{span}\left\{\tilde{g}_{j}, j=1,2, \cdots, m, j \neq i\right\}\right\rangle
$$

$\tilde{g}_{i}(\bar{x})$ and $\bar{g}_{i}(\bar{x})$ denote the $i$ th column of $\tilde{g}(\bar{x})$ and $\bar{g}(\bar{x})$, respectively, and, as usual, $\left\langle\tau_{1}, \tau_{2}, \cdots, \tau_{q} \mid \Delta\right\rangle$ denotes the smallest distribution that contains $\Delta$ and is invariant under the vector fields $\tau_{1}, \tau_{2}, \cdots, \tau_{q}$. Assume that the origin $\bar{x}=0$ is a regular point for $P, P_{i}^{*}, i=1,2, \cdots, m$. It is stressed that the distribution $P$ does not change after a regular state-feedback, i.e.,

$$
P=\left\langle\tilde{f}, \tilde{g}_{1}, \tilde{g}_{2}, \cdots, \tilde{g}_{m} \mid \operatorname{span}\left\{\tilde{g}_{j}, j=1,2, \cdots, m\right\}\right\rangle
$$

moreover, if a suitable set of coordinates $\phi=\left[\phi_{1} \phi_{2}, \cdots, \phi_{\nu}\right]^{T}$ is chosen so that $P^{\perp}=\operatorname{span}\{\mathrm{d} \phi\}$, independently of the choice of the state feedback (13), the subsystem associated with $P^{\perp}$ is described by equations of the form

$$
\dot{\phi}=f_{\phi}(\phi)
$$

i.e., it is not affected at all by the inputs. In the following, the improper notation " $P^{\perp}$ dynamics" will be used to refer to (14), even if, in general, such a subsystem is not associated to any invariant distribution.

Moreover, if $S^{*}$ is the integral submanifold of $P^{*}$ containing the origin $\bar{x}=0$, it has been proven [6, Lemma 7.3.4] that $S^{*}$ is locally invariant under $\tilde{f}(\bar{x})$ and the restriction of $\tilde{f}(\bar{x})$ to $S^{*}$ ( $P^{*}$ dynamics), i.e.,

$$
\dot{\bar{x}}=\left.\tilde{f}(\bar{x})\right|_{S^{*}}
$$

does not depend on the choice of the particular static state-feedback control law (13), provided that noninteraction is achieved.

Finally, let $\Delta_{\text {mix }}$ be the distribution generated by the vector fields $\tau$ defined as in [6, Section 7.4]

$$
\begin{aligned}
\tau=\left[a d_{\tilde{f}}^{k_{q}} \tilde{g}_{i_{q}},\left[\cdots,\left[a d_{\tilde{f}}^{k_{2}} \tilde{g}_{i_{2}}, a d_{\tilde{f}}^{k_{1}} \tilde{g}_{i_{1}}\right]\right]\right], \\
\quad 2 \leq q, \quad k_{i} \geq 0, \quad i_{r} \neq i_{s} \text { for some pair }(r, s) .
\end{aligned}
$$

Let $L^{*}$ denote the integral submanifold of $\Delta_{\text {mix }}$ containing the origin $\bar{x}=0$. The restriction of the closed-loop system to $L^{*}$ ( $\Delta_{\text {mix }}$ dynamics), i.e.,

$$
\dot{\bar{x}}=\left.\tilde{f}(\bar{x})\right|_{L^{*}}
$$

does not depend on the choice of the particular static or dynamic state-feedback control law, provided that noninteraction is achieved (see [6, Proposition 7.4.1]). 


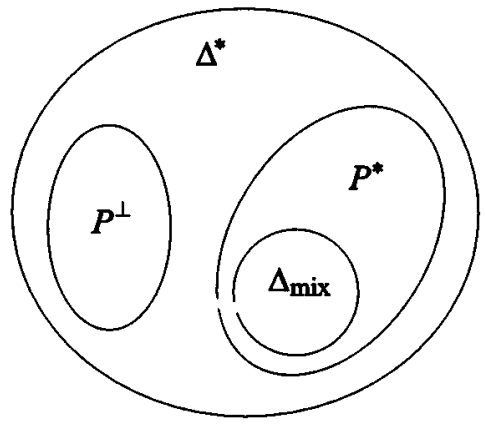

Fig. 1. Qualitative diagram illustrating the inclusion properties relative to dynamics and subsystems concerned with the problem of noninteraction with stability.

To decide which class of control laws has to be used to solve the problem of noninteracting control with stability for system (12), and to decide whether the problem is indeed solvable, the illustrative diagram reported in Fig. 1 can be considered. It clarifies the relations existing between the invariant dynamics and subsystems described above, which are all contained in the zero dynamics of the system (see [6, Ch. 7] for detail). It is clear that, if the subsystem (14) associated with $P^{\perp}$ is not asymptotically stable in the first approximation, a control law yielding a closed-loop system that is stable and noninteractive does not exist. On the other hand, if the zero dynamics of the system are stable in the first approximation, the problem can be solved easily by means of the standard noninteracting feedback of the form (13), composed with a linear "decentralized" state feedback, similar to the one considered in (11) for Hamiltonian systems. With an abuse of notation, such a state feedback will be called in the following "decentralized," or, in the case of simple Hamiltonian systems, in which, as previously stated, $\rho_{i}=1$, "PD-like decentralized." Such a class of state feedback has been used in [5] to solve the problem of noninteracting control with simple stability in the case of Hamiltonian systems having stable zero dynamics. As well known, stability of the zero dynamics is not necessary to solve the noninteracting control problem with stability. As a matter of fact, as shown in [6, Ch. 7] and [2], if general static state feedback control laws are allowed, the problem is solvable if and only if the system itself is stabilizable in the first approximation and the $P^{*}$ dynamics (15) are asymptotically stable in the first approximation. If this last condition is not satisfied, the problem might still be solvable by means of dynamic state feedback. Under some regularity assumptions, in [6] and [2], it is shown that a necessary and sufficient condition for the existence of a dynamic state feedback control law that solves the problem of noninteraction with asymptotic stability in the first approximation is that the system itself is stabilizable in the first approximation and the linearization about the origin of (16) is asymptotically stable. Observe, finally, that for linear systems the distribution $\Delta_{\operatorname{mix}}=\emptyset$. Hence, a linear system can be rendered noninteractive and stable, by means of dynamic state feedback, if and only if the two problems of stabilization and noninteracting control are separately solvable [13], [8], [12]. In particular, the problem of noninteracting control with stability is always solvable for simple linear controllable Hamiltonian systems, with respect to the natural outputs.
Hamiltonian systems are not asymptotically stable at any equilibrium, although they can be stable. As shown in [5], a sufficient condition for stability of a Hamiltonian system is that the Hamiltonian function has an isolated local minimum at the equilibrium. Hence it is of interest to know whether the dynamics of certain subsystems are Hamiltonian, since this fact can highly facilitate the tests for stability, needed, as described above, to decide if the considered problem of noninteracting control with stability is solvable and what class of control laws has to be considered, in order to find a solution.

Despite the fact that the zero dynamics of Hamiltonian systems are Hamiltonian, it will now be shown that, for Hamiltonian systems of the form (1), (2), none of the three subsystems (14)-(16) is Hamiltonian, in general. This will be done by means of simple counterexamples.

Example 1: Consider the following nonlinear system:

$$
\begin{aligned}
& \dot{q}_{1}=p_{1}, \quad \dot{p}_{1}=v_{1}, \quad \dot{q}_{2}=p_{2}, \quad \dot{p}_{2}=v_{2} \\
& \dot{q}_{3}=p_{3}+a_{1} q_{1}+a_{2} q_{2}+\alpha q_{1} q_{2} \\
& \dot{p}_{3}=q_{3}-d_{1} q_{1}-d_{2} q_{2}-\delta q_{1} q_{2}
\end{aligned}
$$

with outputs

$$
\begin{aligned}
& y_{1}=q_{1} \\
& y_{2}=q_{2}
\end{aligned}
$$

obtained by means of the static state-feedback control law

$$
\begin{aligned}
& u_{1}=a_{1} p_{3}+\alpha q_{2} p_{3}+d_{1} q_{3}+\delta q_{2} q_{3}+v_{1} \\
& u_{2}=a_{2} p_{3}+\alpha q_{1} p_{3}+d_{2} q_{3}+\delta q_{1} q_{3}+v_{2}
\end{aligned}
$$

applied to the Hamiltonian system of the form (1), with Hamiltonian function

$$
\begin{aligned}
H(q, p, u)= & \frac{1}{2}\left(p_{1}^{2}+p_{2}^{2}+p_{3}^{2}\right)+\left(a_{1} q_{1}+a_{2} q_{2}+\alpha q_{1} q_{2}\right) p_{3} \\
& -\frac{1}{2} q_{3}^{2}+\left(d_{1} q_{1}+d_{2} q_{2}+\delta q_{1} q_{2}\right) q_{3}-q_{1} u_{1}-q_{2} u_{2}
\end{aligned}
$$

where $a_{i}, d_{i}, \alpha$, and $\delta$ are real parameters of the system. Note that the zero dynamics of such a system can be simply written as

$$
\begin{aligned}
& \dot{q}_{3}=p_{3} \\
& \dot{p}_{3}=q_{3} .
\end{aligned}
$$

We are now ready to prove the following facts.

Fact 1: The $P^{\perp}$ dynamics of Hamiltonian systems of the form (1) need not be Hamiltonian.

Consider system (17). Let $a_{1}=a_{2}=d_{1}=d_{2}=1, \alpha=\delta=$ 0 . Simple calculations show that

$$
P=\operatorname{span}\left\{e_{1}, e_{2}, e_{3}, e_{4}, e_{5}-e_{6}\right\}
$$

Hence, $\phi:=q_{3}+p_{3}$ is such that $P^{\perp}=\operatorname{span}\{\mathrm{d} \phi\}$; the resulting subsystem (14) is

$$
\dot{\phi}=\phi
$$

and it is clearly non-Hamiltonian. Since (19) is unstable, the problem of noninteraction with stability is not solvable. 
Fact 2: The $P^{*}$ dynamics of Hamiltonian systems of the form (1) need not be Hamiltonian.

Consider system (17). Let $a_{1}=d_{1}=a_{2}=\alpha=\delta=1$, $d_{2}=0$ and observe that (locally around the origin)

$$
\begin{aligned}
& P_{1}^{*}=\operatorname{span}\left\{e_{3}, e_{4}, e_{5}, e_{6}\right\} \\
& P_{2}^{*}=\operatorname{span}\left\{e_{1}, e_{2}, e_{5}-e_{6}\right\}
\end{aligned}
$$

whence

$$
P^{*}=P_{1}^{*} \cap P_{2}^{*}=\operatorname{span}\left\{e_{5}-e_{6}\right\} .
$$

As a consequence, $S^{*}=\left\{q_{1}=p_{1}=q_{2}=p_{2}=0, p_{3}=-q_{3}\right\}$ and the dynamics (15) can be written as

$$
\dot{q}_{3}=-q_{3}
$$

which is asymptotically stable (and, obviously, non-Hamiltonian). Moreover, as $P^{\perp}=\emptyset$, the problem of noninteracting control with asymptotic stability in the first approximation can be solved by means of a static state feedback. On the contrary, since the zero dynamics (18) are clearly unstable, the system cannot be rendered noninteractive and stable with a PD-type decentralized control law.

Fact 3: The $\Delta_{\text {mix }}$ dynamics of Hamiltonian systems of the form (1) need not be Hamiltonian.

Consider again system (17), with $a_{1}=d_{1}=a_{2}=\alpha=\delta=$ $1, d_{2}=0$. The vector field $\left[\tilde{g}_{1}, a d_{\tilde{f}}^{3} \tilde{g}_{2}\right]=e_{5}-e_{6}$, belongs to $\Delta_{\text {mix }}$. As $\Delta_{\text {mix }} \subset P^{*}$, it is evident that $\Delta_{\text {mix }} \equiv P^{*}$; hence the $\Delta_{\text {mix }}$ dynamics are not Hamiltonian.

Facts 1-3 have been shown by means of examples in which the relevant dynamics have odd dimension (equal to one). However, this is by no means necessary, as detailed in the following example, related, for brevity, only to Facts 2 and 3.

Example 2: Consider the following nonlinear system:

$$
\begin{aligned}
& \dot{q}_{1}=p_{1}, \quad \dot{p}_{1}=v_{1}, \quad \dot{q}_{2}=p_{2}, \quad \dot{p}_{2}=v_{2} \\
& \dot{q}_{3}=p_{3}+q_{1}+q_{2}+q_{4}+q_{1} q_{2} \\
& \dot{q}_{4}=p_{4}+q_{1}+q_{2}+q_{3} / \sqrt{2}+q_{1} q_{2} \\
& \dot{p}_{3}=q_{3}-q_{1}-2 q_{4}-p_{4} / \sqrt{2}-q_{1} q_{2} \\
& \dot{p}_{4}=2 q_{4}-\sqrt{2} q_{1}-2 q_{3}-p_{3}-\sqrt{2} q_{1} q_{2}
\end{aligned}
$$

with outputs

$$
\begin{aligned}
& y_{1}=q_{1} \\
& y_{2}=q_{2}
\end{aligned}
$$

obtained by means of a suitable static state-feedback control law applied to a Hamiltonian system with Hamiltonian function

$$
\begin{aligned}
H(q, p, u)= & \frac{1}{2}\left(p_{1}^{2}+p_{2}^{2}+p_{3}^{2}+p_{4}^{2}\right)-\frac{1}{2}\left(q_{3}^{2}+2 q_{4}^{2}\right) \\
& +\left(q_{1}+q_{2}+q_{4}+q_{1} q_{2}\right) p_{3}+\left(q_{1}+q_{4}+q_{1} q_{2}\right) q_{3} \\
& +\left(q_{1}+q_{2}+\frac{1}{\sqrt{2}} q_{3}+q_{1} q_{2}\right) p_{4} \\
& +\left(\sqrt{2} q_{1}+q_{3}+\sqrt{2} q_{1} q_{2}\right) q_{4}-q_{1} u_{1}-q_{2} u_{2} .
\end{aligned}
$$

Note that the zero dynamics of such a system can be written as

$$
\begin{aligned}
& \dot{q}_{3}=p_{3}+q_{4} \\
& \dot{q}_{4}=p_{4}+q_{3} / \sqrt{2} \\
& \dot{p}_{3}=q_{3}-2 q_{4}-p_{4} / \sqrt{2} \\
& \dot{p}_{4}=2 q_{4}-2 q_{3}-p_{3} .
\end{aligned}
$$

It is easy to verify that the system described by the last six equations in (20), with $q_{1}=p_{1}=0$, is controllable in the first approximation, with the input $v_{2}$; hence

$$
P_{1}^{*}=\operatorname{span}\left\{e_{3}, e_{4}, e_{5}, e_{6}, e_{7}, e_{8}\right\}
$$

Moreover, after standard computations, one has

$$
P_{2}^{*}=\operatorname{span}\left\{e_{1}, e_{2}, e_{5}-e_{7}, e_{6}-\sqrt{2} e_{8}\right\}
$$

hence

$$
P^{*}=P_{1}^{*} \cap P_{2}^{*}=\operatorname{span}\left\{e_{5}-e_{7}, e_{6}-\sqrt{2} e_{8}\right\}
$$

As a result, $S^{*}$ is given by $\left\{q_{1}=p_{1}=q_{2}=p_{2}=0, p_{3}=\right.$ $\left.-q_{3}, p_{4}=-\sqrt{2} q_{4}\right\}$ and the dynamics (15) can be written, in the coordinates $q_{3}, q_{4}$, as follows:

$$
\begin{aligned}
& \dot{q}_{3}=-q_{3}+q_{4} \\
& \dot{q}_{4}=q_{3} / \sqrt{2}-\sqrt{2} q_{4} .
\end{aligned}
$$

System (21), describing the $P^{*}$ dynamics, is asymptotically stable and is not Hamiltonian.

Finally, it is easy to compute the following vector fields:

$$
\begin{aligned}
& {\left[\tilde{g}_{2}, a d_{\tilde{f}}^{3} \tilde{g}_{1}\right]=\left[\begin{array}{llllllll}
0 & 0 & 0 & 0 & 1 & 1 & -1 & -\sqrt{2}
\end{array}\right]^{T}} \\
& {\left[\tilde{g}_{2}, a d_{\tilde{f}}^{4} \tilde{g}_{1}\right]=\left[\begin{array}{llllllll}
0 & 0 & 0 & 0 & 0 & \sqrt{2} & 0 & -2
\end{array}\right]^{T}}
\end{aligned}
$$

which certainly belong to $\Delta_{\text {mix }}$. As $\Delta_{\text {mix }} \subset P^{*}$, it is evident that $\Delta_{\operatorname{mix}} \equiv P^{*}$, hence the $\Delta_{\operatorname{mix}}$ dynamics are not Hamiltonian.

Remark 1: It must be noted that the Hamiltonian systems considered in Examples 1 and 2 are not simple. The problem of determining if the stronger structure of simple Hamiltonian systems implies that their $P^{\perp}, P^{*}$, or $\Delta_{\text {mix }}$ dynamics are Hamiltonian remains open, and is currently under investigation. However, relative to the $P^{*}$ and the $\Delta_{\text {mix }}$ dynamics, which can be seen as the dynamics of a Hamiltonian system (the zero dynamics) restricted to the integral submanifold of suitable nonsingular, involutive, and invariant distributions, the following considerations can be carried out in order to prove that, under some assumptions, the submanifolds on which they are defined are symplectic.

Assume that a simple Hamiltonian system is given as in (10), with the Hessian matrix with respect to $\tilde{q}$ of the function $V\left(\left[\begin{array}{l}0 \\ \tilde{q}\end{array}\right]\right)$ nonsingular at the origin, which is assumed to be an equilibrium point. Let $\Delta$ be a nonsingular and involutive distribution of dimension $2 r, r<\tilde{n}$, with $\tilde{n}$ being the dimension of $\tilde{q}$, which is $f$-invariant. Let $\chi_{1}(\tilde{q}, \tilde{p}), \cdots, \chi_{s}(\tilde{q}, \tilde{p})$ be $s$ smooth functions such that $\left\{\chi_{1}(\tilde{q}, \tilde{p}), \cdots, \chi_{s}(\tilde{q}, \tilde{p}), \dot{\chi}_{1}(\tilde{q}, \tilde{p}), \cdots, \dot{\chi}_{s}(\tilde{q}, \tilde{p})\right\}$ is a set of $2 s$ independent functions, where, as usual

$$
\dot{\chi}_{i}=\frac{\partial \chi_{i}}{\partial \tilde{q}} \dot{\tilde{q}}+\frac{\partial \chi_{i}}{\partial \tilde{p}} \dot{\tilde{p}}
$$

Assume, further, that $\operatorname{span}\{\mathrm{d} \chi, \mathrm{d} \dot{\chi}\}=\Delta^{\perp}$, i.e., let $s=\tilde{n}-r$. Observe that this is true if $\chi(\tilde{q}, \tilde{p})=\chi(\tilde{q})$. 
In such a case, if $\mathrm{d} \chi \in \Delta^{\perp}$, it is easy to show that $\mathrm{d} \dot{\chi} \in \Delta^{\perp}$, and also the independence of the set of functions $\left\{\chi_{1}(\tilde{q}), \cdots, \chi_{s}(\tilde{q}), \dot{\chi}_{1}(\tilde{q}, \tilde{p}), \cdots, \dot{\chi}_{s}(\tilde{q}, \tilde{p})\right\}$ is easily proven, by virtue of the fact that the matrix $G(\tilde{q})$ is nonsingular. Under such assumptions, it can be proven that the $2 s \times 2 s$-dimensional matrix $C(x)$ defined by

$$
\left[\begin{array}{c|c}
\left\{\chi_{i}, \chi_{j}\right\}(x) & \left\{\chi_{i}, \dot{\chi}_{j}\right\}(x) \\
\hline\left\{\dot{\chi}_{i}, \chi_{j}\right\}(x) & \left\{\dot{\chi}_{i}, \dot{\chi}_{j}\right\}(x)
\end{array}\right]
$$

where $\{a, b\}$ denotes the standard Poisson bracket of the functions $a, b$, and is nonsingular at the origin. Hence, by virtue of [10, Remark 12.37], the integral submanifold of $\Delta$ containing $x=0$ is symplectic for all $x$ in a some neighborhood of the origin. This condition is necessary, but not sufficient, for the dynamics of the given system restricted to $\Delta$ to be Hamiltonian.

\section{MAin Result}

In this section, the problem of noninteracting control with stability will be dealt with for the class of simple Hamiltonian systems given by (1)-(3), using dynamic state-feedback control laws.

In order to tackle jointly several problems related with different stability requirements, let the symbol $\mathbb{C}_{g}$ denote the region of the complex plane, symmetric about the real axis, where the eigenvalues of the linear approximation of the closed-loop system are desired to lie. In particular, let $\mathbb{C}_{g}$ denote the closed left half-plane, or the open left half-plane, or the half-plane $\{s \in \mathbb{C}: \operatorname{Re}(s)<-\alpha\}$, if stability, or asymptotic stability in the first approximation, or asymptotic stability with a prescribed rate of convergence $\alpha$, with $\alpha$ being a positive real number, is required, respectively. Moreover, let $\mathbb{C}_{b}:=\mathbb{C}-\mathbb{C}_{g}$.

It is assumed, without loss of generality, because of the considerations in Section II, that a suitable static state-feedback control law, of the form (6), has already been applied to the given Hamiltonian system to achieve noninteraction. Hence, one can start from (9) for the closed-loop system, i.e.,

$$
\begin{aligned}
\dot{\xi}_{1} & =A \xi_{1}+B v_{1}, \quad \dot{\xi}_{2}=A \xi_{2}+B v_{2} \\
\dot{z} & =F z+L \xi_{1}+M \xi_{2}+\vartheta\left(\xi_{1}, \xi_{2}, z\right) \\
y_{1} & =[10] \xi_{1}, \quad y_{2}=[10] \xi_{2}
\end{aligned}
$$

where the vector $z \in \mathbb{R}^{2 n-4}$ is given by $z:=\left[\tilde{q}^{T} \tilde{p}^{T}\right]^{T}, F, L, M$ are real matrices of suitable dimensions, and the vector valued function $\vartheta\left(\xi_{1}, \xi_{2}, z\right)$ is such that

$$
\frac{\partial \vartheta}{\partial \xi_{1}}(0,0,0)=0, \quad \frac{\partial \vartheta}{\partial \xi_{2}}(0,0,0)=0, \quad \frac{\partial \vartheta}{\partial z}(0,0,0)=0 .
$$

In order to restrict the attention to a class of systems that require dynamic state-feedback control laws in order to be rendered stable and noninteractive, the following two assumptions a) and b) are made.

a) The two pairs

$$
\left(\left[\begin{array}{cc}
A & 0 \\
L & F
\end{array}\right],\left[\begin{array}{c}
B \\
0
\end{array}\right]\right) \text { and }\left(\left[\begin{array}{cc}
A & 0 \\
M & F
\end{array}\right],\left[\begin{array}{c}
B \\
0
\end{array}\right]\right)
$$

are controllable. b) $\sigma(F) \cap \mathbb{C}_{b} \neq\{\emptyset\}$, where the symbol $\sigma(\cdot)$ denotes the spectrum of the matrix at argument.

Notice that assumption a) implies that $P^{*} \equiv \Delta^{*} \equiv$ $\operatorname{span}\{(\partial / \partial z)\}$, hence assumption b) implies that the problem of noninteraction with stability is not solvable by means of static state-feedback.

Now, let $V_{g}^{F}, V_{b}^{F}$ denote the two $F$-invariant subspaces of $\mathbb{R}^{2 n-4}$ such that

$$
\begin{aligned}
\mathbb{R}^{2 n-4} & =V_{g}^{F} \oplus V_{b}^{F} \\
\sigma\left(\left.F\right|_{V_{g}^{F}}\right) & \subset \mathbb{C}_{g} \\
\sigma\left(\left.F\right|_{V_{b}^{F}}\right) & \subset \mathbb{C}_{b} .
\end{aligned}
$$

Let a linear coordinate transformation be defined on $P^{*}$ such that if the new coordinates $\tilde{z}$ are given by $\tilde{z}=T z$, then one has

$$
\tilde{F}:=T F T^{-1}=\left[\begin{array}{cc}
F_{g} & 0 \\
0 & F_{b}
\end{array}\right]
$$

with $\sigma\left(F_{g}\right)=\sigma\left(\left.F\right|_{V_{g}^{F}}\right)$ and $\sigma\left(F_{b}\right)=\sigma\left(\left.F\right|_{V_{b}^{F}}\right)$.

Let $\tilde{z}=:\left[z_{g}^{T} z_{b}^{T}\right]^{T}$ be the partition of $\tilde{z}$ corresponding to the block partition of $\tilde{F}$, and, finally, let the vector $\tilde{\vartheta}\left(\xi_{1}, \xi_{2}, \tilde{z}\right)$, defined by

$$
\tilde{\vartheta}\left(\xi_{1}, \xi_{2}, \tilde{z}\right):=T \vartheta\left(\xi_{1}, \xi_{2}, T^{-1} \tilde{z}\right)
$$

be partitioned according to the partition of $\tilde{z}: \tilde{\vartheta}\left(\xi_{1}, \xi_{2}, \tilde{z}\right)=$ $\left[\vartheta_{g}^{T}\left(\xi_{1} \xi_{2}, \tilde{z}\right) \vartheta_{b}^{T}\left(\xi_{1}, \xi_{2}, \tilde{z}\right)\right]^{T}$.

The following assumption c) considerably simplifies the problem.

c) The vector $\vartheta_{b}\left(\xi_{1}, \xi_{2}, \tilde{z}\right)$ is a function of the variables $\xi_{1}$, $\xi_{2}$ only

$$
\begin{aligned}
& \vartheta_{b}\left(\xi_{1}, \xi_{2}, \tilde{z}\right)=: \psi\left(\xi_{1}, \xi_{2}\right), \\
& \quad \forall\left(\xi_{1}, \xi_{2}, \tilde{z}\right) \text { in a neighborhood of }(0,0,0) .
\end{aligned}
$$

The following result provides a condition to solve the noninteracting control problem with stability by means of dynamic state feedback, which is, in general, much easier to check than the necessary and sufficient conditions based on the explicit computation of the distribution $\Delta_{\text {mix }}$. In order to apply the results recalled in Section III, valid for general nonlinear systems, giving the necessary conditions for the existence of a solution, the following technical assumption is introduced.

d) The origin $\left(\xi_{1}, \xi_{2}, z\right)=(0,0,0)$ is a regular point of the distribution $\Delta_{\text {mix }}$ of system (22).

We are now ready to state the main result of this section.

Proposition 1: Under assumptions a)-d), a dynamic state-feedback control law that solves the noninteracting control problem with A) simple stability, B) asymptotic stability in the first approximation, or C) asymptotic stability with a prescribed convergence rate exists only if the following conditions hold in a neighborhood of $\xi_{1}=0, \xi_{2}=0$ (it is recalled that $\left.\xi_{1}=\left[q_{1} \dot{q}_{1}\right]^{T}, \xi_{2}=\left[q_{2} \dot{q}_{2}\right]^{T}\right)$ :

i) $\psi_{\dot{q}_{1} \dot{q}_{2}}\left(\xi_{1}, \xi_{2}\right)=0$;

ii) $\psi_{q_{1} \dot{q}_{2}}\left(\xi_{1}, \xi_{2}\right)=\psi_{q_{2} \dot{q}_{1}}\left(\xi_{1}, \xi_{2}\right)$;

iii) $(1 / 2) F_{b}\left(\psi_{q_{1} \dot{q}_{2}}\left(\xi_{1}, \xi_{2}\right)+\psi_{q_{2} \dot{q}_{1}}\left(\xi_{1}, \xi_{2}\right)\right)+\psi_{q_{1} q_{2}}\left(\xi_{1}\right.$, $\left.\xi_{2}\right)-\dot{q}_{1} \psi_{q_{1} q_{2} \dot{q}_{1}}\left(\xi_{1}, \xi_{2}\right)-\dot{q}_{2} \psi_{q_{1} q_{2} \dot{q}_{2}}\left(\xi_{1}, \xi_{2}\right)=0$. 
In cases B) and C), conditions i)-iii) are also sufficient for the existence of a solution, whereas, in case A), a set of sufficient conditions is given by i)-iii) and the following condition:

iv) the equilibrium of the dynamical system

$$
\dot{z}_{g}=F_{g} z_{g}+\vartheta_{g}\left(0,0,\left[\begin{array}{c}
z_{g} \\
0
\end{array}\right]\right)
$$

is stable.

\section{Proof of Proposition 1}

In view of the proposed notations, and of assumption c), system (22) can be rewritten as follows:

$$
\begin{aligned}
& \dot{\xi}_{1}=A \xi_{1}+B v_{1} \\
& \dot{\xi}_{2}=A \xi_{2}+B v_{2} \\
& \dot{z}_{g}=F_{g} z_{g}+L_{g} \xi_{1}+M_{g} \xi_{2}+\vartheta_{g}\left(\xi_{1}, \xi_{2},\left[\begin{array}{c}
z_{g} \\
z_{b}
\end{array}\right]\right) \\
& \dot{z}_{b}=F_{b} z_{b}+L_{b} \xi_{1}+M_{b} \xi_{2}+\psi\left(\xi_{1}, \xi_{2}\right)
\end{aligned}
$$

where $L_{g}, L_{b}, M_{g}$, and $M_{b}$ are real matrices of suitable dimensions.

The proof of Proposition 1 is organized as follows. First, it is shown that hypotheses i)-iii) are necessary for the existence of a solution of the given control problem. Second, the design procedure of a dynamic state-feedback compensator is outlined, on the basis of the algorithm proposed in [6, Ch. 7]. In a third step, it is shown that, under hypotheses i)-iii), such a compensator solves the problem of noninteracting control with stability in cases B) and C), i.e., when the stability requirement can be checked on the basis of the properties of the linearized system. Last, by means of some results from the center manifold theory [3], it is shown that, under hypotheses i)-iv), the proposed compensator solves the problem in case A).

\section{A. Necessity of $i)-$-iii)}

In order to see that each of conditions i)-iii) is necessary for the existence of a solution, rewrite system (24) in the general form

$$
\dot{\bar{x}}=\tilde{f}(\bar{x})+\tilde{g}_{1} v_{1}+\tilde{g}_{2} v_{2}
$$

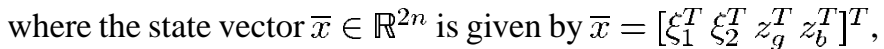
and compute the following vectors, which certainly belong to $\Delta_{\text {mix }}$

$$
\begin{aligned}
{\left[a d_{\tilde{f}} \tilde{g}_{1}, \tilde{g}_{2}\right] } & =\left[\begin{array}{llllll}
0 & 0 & 0 & 0 & l_{g 10}(\bar{x}) & l_{b 10}(\bar{x})
\end{array}\right]^{T} \\
{\left[a d_{\tilde{f}} \tilde{g}_{1}, a d_{\tilde{f}} \tilde{g}_{2}\right] } & =\left[\begin{array}{llllll}
0 & 0 & 0 & 0 & l_{g 11}(\bar{x}) & l_{b 11}(\bar{x})
\end{array}\right]^{T} \\
{\left[a d_{\tilde{f}}^{2} \tilde{g}_{1}, a d_{\tilde{f}} \tilde{g}_{2}\right] } & =\left[\begin{array}{llllll}
0 & 0 & 0 & 0 & l_{g 21}(\bar{x}) & l_{b 21}(\bar{x})
\end{array}\right]^{T}
\end{aligned}
$$

with

$$
\begin{aligned}
l_{b 10}(\bar{x})= & \psi_{\dot{q}_{1} \dot{q}_{2}}\left(\xi_{1}, \xi_{2}\right) \\
l_{b 11}(\bar{x})= & -\psi_{\dot{q}_{1} q_{2}}\left(\xi_{1}, \xi_{2}\right)+\psi_{q_{1} \dot{q}_{2}}\left(\xi_{1}, \xi_{2}\right) \\
l_{b 21}(\bar{x})= & F_{b} \psi_{\dot{q}_{1} q_{2}}\left(\xi_{1}, \xi_{2}\right)-\dot{q}_{2} \psi_{\dot{q}_{1} q_{2} q_{2}}\left(\xi_{1}, \xi_{2}\right) \\
& -\dot{q}_{1} \psi_{q_{1}} \dot{q}_{1} q_{2}\left(\xi_{1}, \xi_{2}\right)+\psi_{q_{1} q_{2}}\left(\xi_{1}, \xi_{2}\right)
\end{aligned}
$$

and with $l_{g 10}(\bar{x}), l_{g 11}(\bar{x})$, and $l_{g 21}(\bar{x})$ being suitable vectors of dimension $n_{g}$. It is clear that, if any of conditions i)-iii) is not satisfied, then $\Delta_{\text {mix }} \cap \operatorname{span}\left\{\left(\partial / \partial z_{b}\right)\right\} \neq 0$, hence the $\Delta_{\text {mix }}$ dynamics of system (24) cannot be stable, with respect to the given stability requirement.

\section{B. Structure of the Overall Control System}

In order to design a dynamic state-feedback compensator, solving the problem of noninteracting control with stability, consider the following nonsingular coordinates transformation:

$$
\tilde{z}_{b}=z_{b}+\tau\left(q_{1}, q_{2}\right)
$$

in which the vector $\tau\left(q_{1}, q_{2}\right)$ is defined as

$$
\begin{aligned}
\tau\left(q_{1}, q_{2}\right):= & \frac{1}{2} \int_{0}^{q_{2}} \int_{0}^{q_{1}}\left(\psi_{q_{1} \dot{q}_{2}}\left(\left[\begin{array}{c}
\eta_{1} \\
\dot{q}_{1}
\end{array}\right],\left[\begin{array}{c}
\eta_{2} \\
\dot{q}_{2}
\end{array}\right]\right)\right. \\
& \left.+\psi_{q_{2} \dot{q}_{1}}\left(\left[\begin{array}{c}
\eta_{1} \\
\dot{q}_{1}
\end{array}\right],\left[\begin{array}{c}
\eta_{2} \\
\dot{q}_{2}
\end{array}\right]\right)\right) \mathrm{d} \eta_{1} \mathrm{~d} \eta_{2} .
\end{aligned}
$$

In the new coordinates, system (24) is described by (24a)-(24c) and

$$
\dot{\tilde{z}}_{b}=F_{b} \tilde{z}_{b}+L_{b} \xi_{1}+M_{b} \xi_{2}+\tilde{\psi}\left(\xi_{1}, \xi_{2}\right)
$$

where, by virtue of hypotheses i)-iii) and of (25), the vector $\tilde{\psi}\left(\xi_{1}, \xi_{2}\right)$ can be seen to satisfy the following four identities:

$$
\begin{aligned}
& \tilde{\psi}_{q_{1} q_{2}}\left(\xi_{1}, \xi_{2}\right)=0, \quad \tilde{\psi}_{q_{1} \dot{q}_{2}}\left(\xi_{1}, \xi_{2}\right)=0 \\
& \tilde{\psi}_{\dot{q}_{1} q_{2}}\left(\xi_{1}, \xi_{2}\right)=0, \quad \tilde{\psi}_{\dot{q}_{1} \dot{q}_{2}}\left(\xi_{1}, \xi_{2}\right)=0
\end{aligned}
$$

in a neighborhood of $\xi_{1}=0, \xi_{2}=0$. This implies that the vector $\tilde{\psi}\left(\xi_{1}, \xi_{2}\right)$ can be written as follows:

$$
\tilde{\psi}\left(\xi_{1}, \xi_{2}\right)=\tilde{\psi}_{1}\left(\xi_{1}\right)+\tilde{\psi}_{2}\left(\xi_{2}\right)
$$

whence, it is easy to verify that $\Delta_{\operatorname{mix}} \subset \operatorname{span}\left\{\left(\partial / \partial z_{g}\right)\right\}$.

Therefore, on the basis of the synthesis procedure reported in [6, Section 7.5], valid for general nonlinear systems, it is possible to design a dynamic state-feedback compensator for the subsystem

$$
\begin{aligned}
& \dot{\xi}_{1}=A \xi_{1}+B v_{1} \\
& \dot{\xi}_{2}=A \xi_{2}+B v_{2} \\
& \dot{\tilde{z}}_{b}=F_{b} \tilde{z}_{b}+L_{b} \xi_{1}+M_{b} \xi_{2}+\tilde{\psi}_{1}\left(\xi_{1}\right)+\tilde{\psi}_{2}\left(\xi_{2}\right)
\end{aligned}
$$

which solves the problem of noninteraction with asymptotic stability in the first approximation [in both cases A) and B)], or with the desired convergence rate [in case C)]. Such a compensator is of the form

$$
\begin{aligned}
\dot{x}_{c} & =\gamma\left(\xi_{1}, \xi_{2}, \tilde{z}_{b}, x_{c}\right)+G_{c} w \\
v & =\eta\left(\xi_{1}, \xi_{2}, \tilde{z}_{b}, x_{c}\right)+w
\end{aligned}
$$

where $x_{c}$ is the state vector, $x_{c} \in \mathbb{R}^{4+2} n_{b}$, with $n_{b}:=\operatorname{dim}\left(V_{b}^{F}\right)$, and $w$ is the vector of the new inputs, $w=\left[\begin{array}{ll}w_{1} & w_{2}\end{array}\right]^{T}$, where $w_{1}$ does not affect $y_{2}$ and $w_{2}$ does not affect $y_{1}$.

Now, letting $x_{e}:=\left[\begin{array}{llll}\xi_{1}^{T} & \xi_{2}^{T} & z_{b}^{T} & x_{c}^{T}\end{array}\right]^{T}$, the closed-loop system (26), (27) can be written as

$$
\begin{aligned}
& \dot{x}_{e}=f_{e}\left(x_{e}\right)+g_{1 e}\left(x_{e}\right) w_{1}+g_{2 e}\left(x_{e}\right) w_{2} \\
& y_{1}=h_{1 e}\left(x_{e}\right) \\
& y_{2}=h_{2 e}\left(x_{e}\right)
\end{aligned}
$$


and, in view of the design procedure adopted, it is noninteractive and asymptotically stable in the first approximation [with the desired convergence rate, in case $\mathrm{C}$ )].

\section{Sufficiency of $i$ )-iii) in Cases B) and C)}

Simple considerations relative to the linearization about the origin of the overall control system, constituted by (28) and by

$\dot{z}_{g}=F_{g} z_{g}+L_{g} \xi_{1}+M_{g} \xi_{2}+\vartheta_{g}\left(\xi_{1}, \xi_{2},\left[\begin{array}{c}z_{g} \\ \tilde{z}_{b}-\tau\left(q_{1}, q_{2}\right)\end{array}\right]\right)$

suffice to prove the proposition with respect to the stability requirements B) and C), in view of the fact that the outputs $y_{1}$ and $y_{2}$ are not affected by $z_{g}$.

\section{Sufficiency of $i$ )-iv) in Case A)}

The stability of the origin $x_{e}=0, z_{g}=0$, for the overall control system, composed of (28) and (29), can be proven by means of well-known results from the center manifold theory (see [3] and [6, Appendix B]). To this end, consider a change of coordinates on the state space of system (23) such that, if the new coordinates $\tilde{z}_{g}$ are $\tilde{z}_{g}=\tilde{T} z_{g}$, then one has

$$
\tilde{F}_{g}:=\tilde{T} F_{g} \tilde{T}^{-1}=\left[\begin{array}{cc}
F_{g-} & 0 \\
0 & F_{g 0}
\end{array}\right]
$$

with $\sigma\left(F_{g-}\right) \subset\{\lambda \in \mathbb{C}, \operatorname{Re}(\lambda)<0\}$ and $\sigma\left(F_{g 0}\right) \subset\{\lambda \in$ $\mathbb{C}, \operatorname{Re}(\lambda)=0\}$.

Let $\tilde{z}_{g}=\left[z_{g-}^{T} z_{g 0}^{T}\right]^{T}$ be the partition of $\tilde{z}_{g}$ corresponding to the block partition of $\tilde{F}_{g}$. In the new coordinates, system (23) can be rewritten as

$$
\begin{gathered}
\dot{z}_{g-}=F_{g-} z_{g-}+\vartheta_{g-}\left(z_{g-}, z_{g 0}\right) \\
\dot{z}_{g 0}=F_{g 0} z_{g 0}+\vartheta_{g 0}\left(z_{g-}, z_{g 0}\right)
\end{gathered}
$$

with

$$
\begin{aligned}
\vartheta_{g-}\left(z_{g-}, z_{g 0}\right) & :=\tilde{T}_{-} \vartheta_{g}\left(0,0,\left[\begin{array}{c}
\left.\tilde{T}^{-1}\left[\begin{array}{c}
z_{g-} \\
z_{g 0}
\end{array}\right]\right) \\
0
\end{array}\right]\right) \\
\vartheta_{g 0}\left(z_{g-}, z_{g 0}\right) & :=\tilde{T}_{0} \vartheta_{g}\left(0,0,\left[\begin{array}{c}
\left.\tilde{T}^{-1}\left[\begin{array}{c}
z_{g-} \\
z_{g 0}
\end{array}\right]\right) \\
0
\end{array}\right]\right)
\end{aligned}
$$

where $\tilde{T}_{-}, \tilde{T}_{0}$ are the two row blocks of the partition $\tilde{T}=$ $\left[\begin{array}{c}\tilde{T}_{-} \\ \tilde{T}_{0}\end{array}\right]$ of matrix $\tilde{T}$, corresponding to the block partition of $\tilde{F}_{g}$. Since the origin $z_{g}=0$ is an equilibrium point of system (23), then it is clear that functions $\vartheta_{g-}(\cdot, \cdot)$ and $\vartheta_{g 0}(\cdot, \cdot)$ vanish at $\left[\begin{array}{c}z_{g-} \\ z_{g 0}\end{array}\right]=\left[\begin{array}{l}0 \\ 0\end{array}\right]$, hence a mapping $z_{g-}=\pi\left(z_{g 0}\right)$, defined on a neighborhood $U$ of $z_{g 0}=0$, such that the set

$$
S:=\left\{\left[\begin{array}{c}
z_{g-} \\
z_{g 0}
\end{array}\right] \in \mathbb{R}^{n_{g}}: z_{g 0} \in U, z_{g-}=\pi\left(z_{g 0}\right)\right\}
$$

is a center manifold for system (30), exists. Moreover, by hypothesis iv), it follows that the dynamics of system (30) restricted to $S$, described by the equation

$$
\dot{z}_{g 0}=F_{g 0} z_{g 0}+\vartheta_{g 0}\left(\pi\left(z_{g 0}\right), z_{g 0}\right), \quad z_{g 0} \in U
$$

are necessarily stable. Now, in order to see that this implies stability for the overall control system, which can be written as

$$
\begin{aligned}
\dot{x}_{e} & =f_{e}\left(x_{e}\right)+g_{1 e} v_{1}+g_{2 e} v_{2} \\
\dot{z}_{g-} & =F_{g-} z_{g-}+L_{-} x_{e}+\tilde{\vartheta}_{g-}\left(x_{e}, z_{g-}, z_{g 0}\right) \\
\dot{z}_{g 0} & =F_{g 0} z_{g 0}+L_{0} x_{e}+\tilde{\vartheta}_{g 0}\left(x_{e}, z_{g-}, z_{g 0}\right)
\end{aligned}
$$

where the matrices $L_{-}, L_{0}$ take into account the terms linear in $\xi_{1}, \xi_{2}$ appearing in $(24 \mathrm{c})$, and

$$
\begin{aligned}
& \tilde{\vartheta}_{g-}\left(x_{e}, z_{g-}, z_{g 0}\right):=\tilde{T}_{-\vartheta_{g}}\left(\xi_{1}, \xi_{2},\left[\begin{array}{c}
\left.\tilde{T}^{-1}\left[\begin{array}{c}
z_{g-} \\
z_{g 0}
\end{array}\right]\right) \\
\tilde{z}_{b}-\tau\left(q_{1}, q_{2}\right)
\end{array}\right]\right) \\
& \tilde{\vartheta}_{g 0}\left(x_{e}, z_{g-}, z_{g 0}\right):=\tilde{T}_{0} \vartheta_{g}\left(\xi_{1}, \xi_{2},\left[\begin{array}{c}
\tilde{T}^{-1}\left[\begin{array}{c}
z_{g-} \\
z_{g 0}
\end{array}\right] \\
\tilde{z}_{b}-\tau\left(q_{1}, q_{2}\right)
\end{array}\right]\right)
\end{aligned}
$$

a further linear coordinates transformation is needed. Define

$$
F_{e}:=\left.\frac{\partial f_{e}\left(x_{e}\right)}{\partial x_{e}}\right|_{x_{e}=0}
$$

and let the last vector component of the new coordinates vector $\left[x_{e}^{T} z_{g-}^{T} \tilde{z}_{g 0}^{T}\right]^{T}$ be given by

$$
\tilde{z}_{g 0}=P_{0} x_{e}+z_{g 0}
$$

where the matrix $P_{0} \in \mathbb{R}^{\left(2 n-4-2 n_{b}\right) \times\left(8+3 n_{b}\right)}$ is such that

$$
F_{g 0} P_{0}-P_{0} F_{e}=L .
$$

The existence of such a matrix $P_{0}$ is guaranteed by the fact that $\sigma\left(F_{g 0}\right) \cap \sigma\left(F_{e}\right)=\{\emptyset\}$. System (32), with $v_{1}=v_{2}=0$, can be rewritten as follows:

$$
\begin{aligned}
\dot{x}_{e} & =F_{e} x_{e}+\Delta f_{e}\left(x_{e}\right) \\
\dot{z}_{g-} & =F_{g-} z_{g-}+L_{-} x_{e}+\tilde{\vartheta}_{g-}\left(x_{e}, z_{g-}, \tilde{z}_{g 0}-P_{0} x_{e}\right) \\
\dot{\tilde{z}}_{g 0} & =\Delta f_{e}^{0}\left(x_{e}\right)+F_{g 0} \tilde{z}_{g 0}+\tilde{\vartheta}_{g 0}\left(x_{e}, z_{g-}, \tilde{z}_{g 0}-P_{0} x_{e}\right)
\end{aligned}
$$

where the functions $\Delta f_{e}\left(x_{e}\right):=f_{e}\left(x_{e}\right)-F_{e} x_{e}$ and $\Delta f_{e}^{0}\left(x_{e}\right):=P_{0} f_{e}\left(x_{e}\right)-F_{g 0} P_{0} x_{e}+L_{0} x_{e}$ vanish, together with their Jacobian matrices with respect to $x_{e}$, at $x_{e}=0$.

It is easy to see that the set

$$
\begin{gathered}
S_{e}:=\left\{\left[\begin{array}{c}
x_{e} \\
z_{g-} \\
\tilde{z}_{g 0}
\end{array}\right] \in \mathbb{R}^{\left(8+3 n_{b}+n_{g}\right)}: \tilde{z}_{g 0} \in U,\right. \\
\left.x_{e}=0, z_{g-}=\pi\left(\tilde{z}_{g 0}\right)\right\}
\end{gathered}
$$

is a center manifold for system (33), and that the dynamics of system (33) restricted to $S_{e}$ coincide with (31) if $z_{g 0}$ is replaced by $\tilde{z}_{g 0}$. The claim follows from the reduction principle [3].

\section{EXAMPLES}

In this section, two examples, stemming from simple mechanical systems, are presented, and the application of the theory reported in Sections II-IV is discussed. 


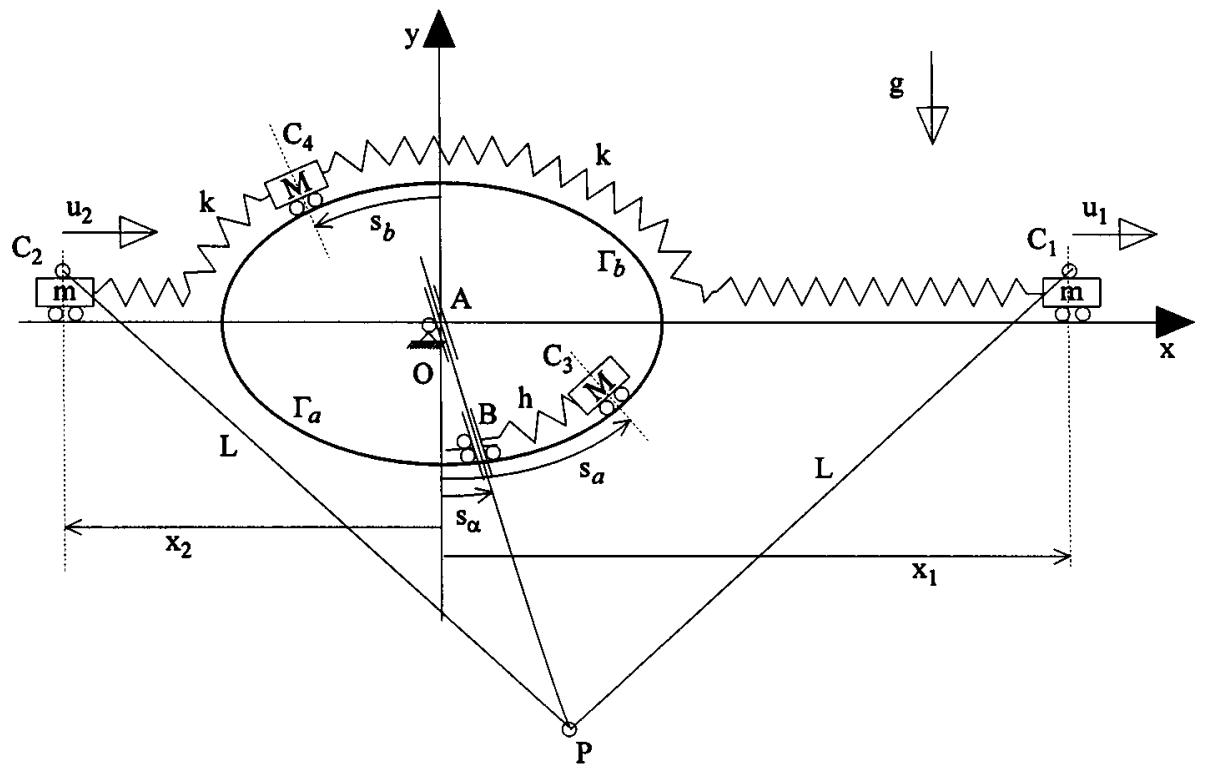

Fig. 2. The mechanical system considered in Example 3.

The first example is an unstable system, for which noninteraction and simple stability are jointly achievable, if the use of dynamic state-feedback control is allowed.

Example 3: Consider the system represented in Fig. 2, which is composed of four heavy dimensionless carts, denoted by $C_{1}$, $C_{2}, C_{3}$, and $C_{4}$, which are subject to the gravitational field, of magnitude $g$, and are constrained to move along specified curves lying on a vertical plane. The four carts, $C_{1}$ and $C_{2}$ having mass $m, C_{3}$ and $C_{4}$ having mass $M$, interact between them through mechanical couplings involving other massless objects, as described in the following. Any kind of friction is neglected in the proposed model of the system.

On the vertical plane an inertial reference frame $x O y$ is defined, whose $y$-axis is parallel to the gravity acceleration vector and has opposite direction.

The two carts $C_{3}$ and $C_{4}$ are constrained to slide along two curves, $\Gamma_{a}$ and $\Gamma_{b}$, each parameterized through its curvilinear abscissa $s \in[-2,2]$ :

$$
\begin{aligned}
& \Gamma_{a}:\left\{\begin{array}{l}
x_{a}(s)=\frac{s}{2} \sqrt{1-\frac{s^{2}}{4}}+\arcsin \left(\frac{s}{2}\right) \\
y_{a}(s)=-1+\frac{s^{2}}{4}
\end{array}\right. \\
& \Gamma_{b}:\left\{\begin{array}{l}
x_{b}(s)=-\frac{s}{2} \sqrt{1-\frac{s^{2}}{4}}-\arcsin \left(\frac{s}{2}\right) \\
y_{b}(s)=1-\frac{s^{2}}{4} .
\end{array}\right.
\end{aligned}
$$

The cart $C_{3}$ slides along $\Gamma_{a}$; hence its position at time $t$ is $\left(x_{a}\left(s_{a}(t)\right), y_{a}\left(s_{a}(t)\right)\right)$ and $s_{a}(t)$ can be taken as its configuration coordinate; similarly, the position of the cart $C_{4}$, which slides along $\Gamma_{b}$, is $\left(x_{b}\left(s_{b}(t)\right), y_{b}\left(s_{b}(t)\right)\right)$, and $s_{b}(t)$ can be taken as its configuration coordinate.

The two carts $C_{1}$ and $C_{2}$ slide along the $x$-axis, so that their configuration coordinates can be simply taken as $x_{1}$ and $x_{2}$.
Carts $C_{1}$ and $C_{2}$ are subject to two external forces having direction parallel to the $x$-axis and intensity $u_{1}$ and $u_{2}$, respectively (the only control inputs). Two linear, elastic, massless springs, having length $L_{0}>2$ when undeformed, and elastic constant $k$ connect the cart $C_{4}$ with the carts $C_{1}$ and $C_{2}$; as shown in Fig. 2, such springs lie on the same curves along which $C_{1}, C_{2}$, and $C_{4}$ are constrained to slide.

A massless cylinder, denoted with $B$ in Fig. 2, whose axis belongs to the plane $x O y$, is free to rotate about a hinge, whose axis is perpendicular to the plane $x O y$, placed on a massless cart, which is constrained to slide along $\Gamma_{a}$; the dimensions of $B$ of the hinge and of the cart are all negligible, so that those three objects can be considered as a single point, whose position is $\left(x_{a}\left(s_{\alpha}(t)\right), y_{a}\left(s_{\alpha}(t)\right)\right)$, with $s_{\alpha}(t)$ being the value of the curvilinear abscissa of such a point on $\Gamma_{a}$. A linear, elastic, massless spring, having length equal to zero when undeformed, and elastic constant $h$ connects $C_{3}$ with the cart on which $B$ is hinged; such a spring is also constrained to lie on $\Gamma_{a}$. A further mechanical coupling is established between $C_{1}$ and $C_{2}$ and the cart supporting $B$, by means of three massless rigid bars, also lying on the plane $x O y$, which are hinged at one extremity with the hinge $P$. Two of the bars, having length $L>\sqrt{1+\left(L_{0}-2+\pi / 2\right)^{2}}$, are hinged at the other extremity, one at each of the carts $C_{1}$ and $C_{2}$. The third bar, whose length is not relevant, provided that it is greater than the maximum distance between the hinge $P$ and the origin $O$, is constrained by means of two prismatic one-degree-of-freedom couplings, the first with the cylinder $B$ and the other with a second dimensionless and massless cylinder, denoted by $A$. The axis $A$ also belongs to the plane $x O y$, and, by means of a dimensionless hinge placed at $O$, it is assured that the central point of $A$ coincides with the origin $O$.

The described interaction between the carts $C_{1}$ and $C_{2}$, the cylinders $A$ and $B$, and the three bars hinged at $P$ guarantees that the curvilinear abscissa $s_{\alpha}(t)$ of $B$ along $\Gamma_{a}$ is a function of the position coordinates $x_{1}(t)$ and $x_{2}(t)$ of $C_{1}$ and $C_{2}$. The 
function $s_{\alpha}=s_{\alpha}\left(x_{1}, x_{2}\right)$ is defined implicitly by means of the equation $F\left(x_{1}, x_{2}, s_{\alpha}\right)=0$, where

$$
\begin{aligned}
& F\left(x_{1}, x_{2}, s_{\alpha}\right) \\
& \quad:=x_{a}\left(s_{\alpha}\right) \sqrt{L^{2}-\left(\frac{x_{1}-x_{2}}{2}\right)^{2}}+y_{a}\left(s_{\alpha}\right) \frac{x_{1}+x_{2}}{2}
\end{aligned}
$$

in the domain of interest: $x_{1}>\pi / 2, x_{2}<-\pi / 2, x_{1}-x_{2}<2 L$, $-2<s_{\alpha}<2$.

The system is in equilibrium if $x_{1}=x_{e}, x_{2}=-x_{e}, s_{a}=$ $s_{b}=0$, where $x_{e}:=L_{0}-2+\pi / 2$. Therefore, a suitable vector of configuration coordinates is $q=\left[x_{1}-x_{e} x_{2}+x_{e} s_{a} s_{b}\right]^{T}$; in the following, the motion of the system around the origin $q=0$ is considered.

The kinetic and potential energies of the system can be written as follows:

$$
\begin{aligned}
T(\dot{q})= & \frac{1}{2}\left(m\left(\dot{q}_{1}^{2}+\dot{q}_{2}^{2}\right)+M\left(\dot{q}_{3}^{2}+\dot{q}_{4}^{2}\right)\right) \\
V(q)= & M g\left(y_{a}\left(q_{3}\right)+y_{b}\left(q_{4}\right)\right) \\
& +\frac{1}{2}\left(k\left(q_{1}+q_{4}\right)^{2}+k\left(q_{2}+q_{4}\right)^{2}\right. \\
& \left.\quad+h\left(q_{3}-s_{\alpha}\left(q_{1}+x_{e}, q_{2}-x_{e}\right)\right)^{2}\right) .
\end{aligned}
$$

Using (34) and (35) and the discussion in [10, Ch. 12], it follows that

$$
H_{0}(q, p)=T\left(\left[\begin{array}{llll}
p_{1} / m & p_{2} / m & p_{3} / M & p_{4} / M
\end{array}\right]^{T}\right)+V(q)
$$

in which $p=\left[\begin{array}{llll}m \dot{q}_{1} & m \dot{q}_{2} & M \dot{q}_{3} & M \dot{q}_{4}\end{array}\right]^{T}$. As a result, the state space equations describing the system can be obtained as in Section II:

$$
\begin{aligned}
\ddot{q}_{1} & =\frac{1}{m}\left(\bar{f}_{2}(q)+u_{1}\right) \\
\ddot{q}_{2} & =\frac{1}{m}\left(\bar{f}_{4}(q)+u_{2}\right) \\
\dot{q}_{3} & =\frac{1}{M} p_{3} \\
\dot{q}_{4} & =\frac{1}{M} p_{4} \\
\dot{p}_{3} & =h\left(s_{\alpha}\left(q_{1}+x_{e}, q_{2}-x_{e}\right)-q_{3}\right)-\frac{M g}{2} q_{3} \\
\dot{p}_{4} & =-k\left(q_{1}+q_{2}+2 q_{4}\right)+\frac{M g}{2} q_{4}
\end{aligned}
$$

with the natural outputs

$$
\begin{aligned}
& y_{1}=q_{1} \\
& y_{2}=q_{2}
\end{aligned}
$$

The functions $\bar{f}_{2}(\cdot)$ and $\bar{f}_{4}(\cdot)$ are given by

$$
\begin{aligned}
& \bar{f}_{2}(q)=-k\left(q_{1}+q_{4}\right)-\frac{h\left(q_{3}-s_{\alpha}\right)\left(-4+s_{\alpha}^{2}-n(q)\right)}{2 d(q)} \\
& \bar{f}_{4}(q)=-k\left(q_{2}+q_{4}\right)-\frac{h\left(q_{3}-s_{\alpha}\right)\left(-4+s_{\alpha}^{2}+n(q)\right)}{2 d(q)}
\end{aligned}
$$

where the dependence of $s_{\alpha}$ on $q$ is omitted, and

$$
\begin{aligned}
& n(q)=\frac{\left(q_{1}-q_{2}+2 x_{e}\right)\left(s_{\alpha} \sqrt{4-s_{\alpha}^{2}}+4 \arcsin \left(\frac{s_{\alpha}}{2}\right)\right)}{\sqrt{4 L^{2}-\left(q_{1}-q_{2}+2 x_{e}\right)^{2}}} \\
& d(q)=\left(q_{1}+q_{2}\right) s_{\alpha}+\sqrt{4-s_{\alpha}^{2}} \sqrt{4 L^{2}-\left(q_{1}-q_{2}+2 x_{e}\right)^{2}} .
\end{aligned}
$$

After a first state feedback of the form

$$
\begin{aligned}
& u_{1}=-\bar{f}_{2}(q)+m v_{1} \\
& u_{2}=-\bar{f}_{4}(q)+m v_{2}
\end{aligned}
$$

if $\xi_{1}$ and $\xi_{2}$ are given by (8), the system (36), (37) can be written in the form (22), with $z=\left[\begin{array}{llll}q_{3} & q_{4} & p_{3} & p_{4}\end{array}\right]^{T}$. It is easy to see that assumption a) holds. In order to study the stability properties, in the first approximation of the zero dynamics, the matrix $F$ has to be considered

$$
F=\left[\begin{array}{cccc}
0 & 0 & 1 / M & 0 \\
0 & 0 & 0 & 1 / M \\
-h-g M / 2 & 0 & 0 & 0 \\
0 & -2 k+g M / 2 & 0 & 0
\end{array}\right]
$$

If $k<(g M / 4)$, the matrix $F$ has a real eigenvalue $\lambda$ with positive real part, hence stability of the closed-loop system cannot be achieved by any static state-feedback control law that guarantees noninteraction. Since the eigenvalues of $F$ are $\{\lambda,-\lambda, \chi \omega,-\chi \omega\}$, with $\lambda=(1 / \sqrt{2}) \sqrt{g-(4 k / M)}$ and $\omega=(1 / \sqrt{2}) \sqrt{g+(2 h / M)}$, and $\jmath$ being the imaginary unit, it makes sense to check for the existence of a dynamic state-feedback control law guaranteeing noninteraction and simple stability. The closed-loop system (36), (37) can be put in the form (24) by means of a coordinates transformation

$$
\tilde{z}=T z
$$

where the matrix $T$ is such that

$$
\tilde{F}=T F T^{-1}=\left[\begin{array}{rrrr}
\Lambda_{1} & 0 & 0 \\
0 & 0 & -\lambda & 0 \\
0 & 0 & 0 & \lambda
\end{array}\right]
$$

with $\sigma\left(\Lambda_{1}\right)=\{-\not \omega, \jmath \omega\}$. After such a transformation, it turns out that $(24 d)$ is given by

$$
\dot{z}_{b}=k_{1} q_{1}+k_{2} q_{2}+\lambda z_{b}, \quad k_{1}, k_{2} \in \mathbb{R}
$$

hence $\psi\left(\xi_{1}, \xi_{2}\right)=0$ and hypotheses i)-iii) are satisfied. In order to check that iv) also holds, it is sufficient to notice that, by letting $\xi_{1}=\xi_{2}=0$, the zero dynamics of the system are given by two decoupled subsystems, the first describing the dynamics of the cart $C_{3}$ and the second the ones of $C_{4}$. As for cart $C_{3}$, when $\xi_{1}=\xi_{2}=0$, its motion is described by (36c) and (36e) with $s_{\alpha}\left(x_{e},-x_{e}\right)=0$

$$
\begin{aligned}
& \dot{q}_{3}=p_{3} / M \\
& \dot{p}_{3}=-(h+M g / 2) q_{3}
\end{aligned}
$$




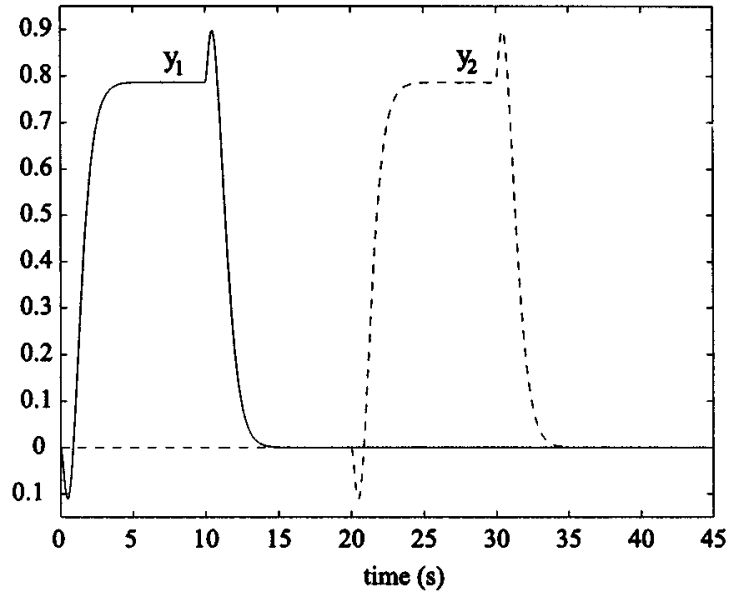

(a)

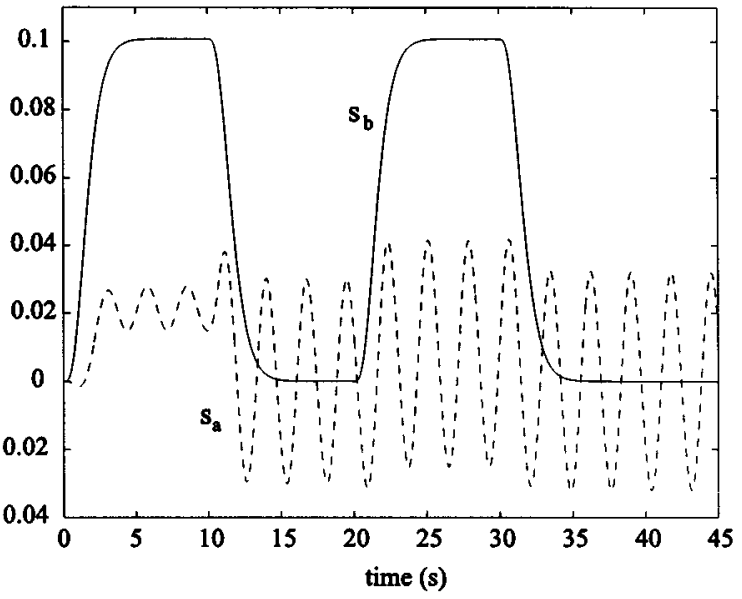

(b)

Fig. 3. Simulation results for the noninteracting control system obtained in Example 3: time behavior of (a) the two outputs $y_{1}$ and $y_{2}$ and (b) the nonactuated state variables $s_{a}$ and $s_{b}$.

from which it is evident that such a subsystem is simply stable. As for cart $C_{4}$, its constrained dynamics

$$
\begin{aligned}
\dot{q}_{b} & =p_{4} / M \\
\dot{p}_{4} & =(-2 k+M g / 2) q_{4}
\end{aligned}
$$

are clearly split by the coordinates transformation (38) into the unstable part, given by (39) with $q_{1}=q_{2}=0$ and an analogous equation relative to an asymptotically stable subsystem. It follows that hypothesis iv) is satisfied, with the components of $z_{g}$ being $q_{3}, p_{3}$ and the state variable of the asymptotically stable subsystem just mentioned.

On the basis of the synthesis techniques reported in [6, Section 7.5], a dynamic state-feedback compensator, of the form (27), has been designed in order to render the overall closed-loop system simply stable and noninteractive. The results of a significant simulation of the behavior of the overall control system are reported in Fig. 3. For the simulation reported here, the values of the physical parameters have been taken as follows: $g=9.81, m=1, M=2, k=1, h=1 / 2$, $L_{0}=5-\pi / 2, L=3.5$. Starting from initial conditions equal to zero for all the state variables, two piece-wise constant input functions $w_{1}(\cdot)$ and $w_{2}(\cdot)$ have been applied, one at each of the two decoupled channels: $w_{1}(t)$ different from zero only for $t \in[0,10]$ and $w_{2}(t)$ different from zero only for $t \in[20,30]$. In Fig. 3(a), one can see that each output $y_{i}$ is not affected by the values of the input function $w_{j}, j \neq i$, whereas in Fig. 3(b), it is possible to appreciate the time behavior of the positions of two carts $C_{3}$ and $C_{4}$.

The next example is concerned with a simple physical system, which can be rendered noninteractive and asymptotically stable in the first approximation by means of a suitable static state-feedback control law, although its zero dynamics are not trivial and not asymptotically stable.

Example 4: Consider the system represented in Fig. 4, which is constituted by three equal bodies having mass $m$ each, which slide along an horizontal axis, namely, the $x$-axis of some inertial reference frame. Any kind of friction is neglected in the

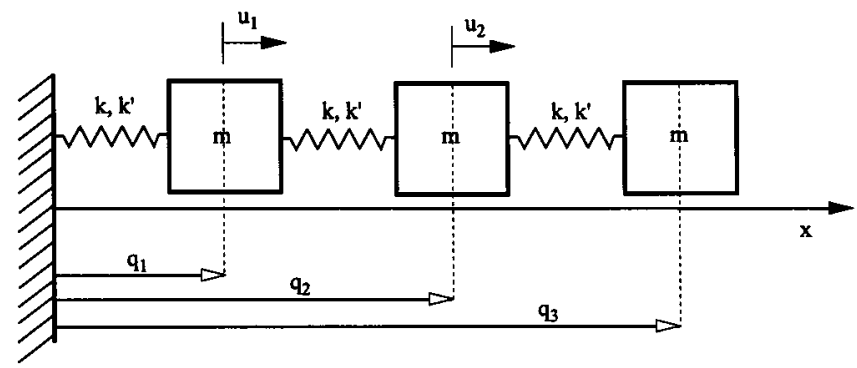

Fig. 4. The mechanical system considered in Example 4.

proposed model of the system. The first body, whose position at time $t$ is denoted by $q_{1}(t)$, is connected to the origin by means of a nonlinear elastic spring (so-called hardening spring), having length equal to zero, when undeformed, and exerting a force equal in modulus to

$$
F(\ell)=k \ell+k^{\prime} \ell^{3}
$$

on the bodies at its extremities, when deformed up to length $\ell$. The second body, whose position is denoted by $q_{2}(t)$, is connected to the first one by means of a nonlinear elastic spring equal to the one described previously, whereas the third body, whose position is denoted by $q_{3}(t)$, is connected in the same way to the second one. The two control inputs of the system are two external forces, $u_{1}(t)$ and $u_{2}(t)$, applied to the first and to the second body, respectively. The natural outputs of the system are $y_{1}=q_{1}, y_{2}=q_{2}$.

Since the kinetic and potential energies of the system are given by

$$
\begin{aligned}
T(\dot{q})= & \frac{1}{2} m\left(\dot{q}_{1}^{2}+\dot{q}_{2}^{2}+\dot{q}_{3}^{2}\right) \\
V(q)= & \frac{1}{2} k\left(q_{1}^{2}+\left(q_{2}-q_{1}\right)^{2}+\left(q_{3}-q_{2}\right)^{2}\right) \\
& +\frac{1}{4} k^{\prime}\left(q_{1}^{4}+\left(q_{2}-q_{1}\right)^{4}+\left(q_{3}-q_{2}\right)^{4}\right)
\end{aligned}
$$


the equations of motion can be written as

$$
\begin{gathered}
\ddot{q}_{1}=\frac{1}{m}\left(k\left(-2 q_{1}+q_{2}\right)+k^{\prime}\left(-q_{1}^{3}+\left(q_{2}-q_{1}\right)^{3}\right)+u_{1}\right) \\
\ddot{q}_{2}=\frac{1}{m}\left(k\left(q_{1}-2 q_{2}+q_{3}\right)\right. \\
\left.\quad+k^{\prime}\left(-\left(q_{2}-q_{1}\right)^{3}+\left(q_{3}-q_{2}\right)^{3}\right)+u_{2}\right) \\
\dot{q}_{3}=\frac{1}{m} p_{3}, \quad \dot{p}_{3}=k\left(q_{2}-q_{3}\right)+k^{\prime}\left(q_{2}-q_{3}\right)^{3}
\end{gathered}
$$

where $p_{3}:=m \dot{q}_{3}$.

After a first state feedback (6), the closed-loop system is noninteractive and has the form

$$
\begin{aligned}
& \ddot{q}_{1}=v_{1}, \quad \ddot{q}_{2}=v_{2}, \quad \dot{q}_{3}=\frac{1}{m} p_{3} \\
& \dot{p}_{3}=k\left(x_{2}-q_{3}\right)+k^{\prime}\left(x_{2}-q_{3}\right)^{3} .
\end{aligned}
$$

The zero dynamics of system (40) can be written as

$$
\begin{aligned}
& \dot{q}_{3}=\frac{1}{m} p_{3} \\
& \dot{p}_{3}=-k q_{3}-k^{\prime} q_{3}^{3}
\end{aligned}
$$

since they are not asymptotically stable (they are those of an undamped Duffing oscillator), the approach of [5] cannot succeed in obtaining noninteraction with asymptotic stability. However, it is clear that the distribution $P^{*}$ has zero dimension for system (40), hence the system (which is controllable in the first approximation) can be stabilized asymptotically by means of a static state feedback, which preserves noninteraction. As a matter of fact, any feedback control law described by equations of the form

$$
\begin{aligned}
& v_{1}=-\epsilon_{11} x_{1}-\epsilon_{12} \dot{x}_{1}+w_{1}, \quad \epsilon_{11}>0, \quad \epsilon_{12}>0 \\
& v_{2}=\alpha\left(x_{2}, \dot{x}_{2}, q_{3}, p_{3}\right)+w_{2}
\end{aligned}
$$

which asymptotically stabilizes in the first approximation system (40), succeeds in obtaining a closed-loop system, which is noninteractive (the input $w_{i}$ does not affect the output $y_{j}$, $i \neq j$ ) and asymptotically stable in the first approximation.

\section{CONCLUSIONS}

In this paper, the problem of noninteracting control with stability has been tackled for a class of nonlinear Hamiltonian systems, by means of (possibly) dynamic state feedback control laws. Using well-known results from nonlinear geometric control, it has been possible to enlarge the class of systems proposed in [5], for which the problem of noninteracting control with simple stability is solvable by means of static state feedback. A wider class of systems has been determined for which the problem is solvable if dynamic state feedback is allowed. Mathematical and physical examples have been presented and discussed.
Further work will be devoted to the subject, to enlarge further the class of systems for which the problem of noninteracting control with stability is solvable. Moreover the fine structure of simple Hamiltonian systems is under study, with particular emphasis on Hamiltonian properties of the $P^{\perp}, P^{*}$, and $\Delta_{\text {mix }}$ dynamics.

\section{REFERENCES}

[1] A. Astolfi, "Control of hamiltonian systems," in Modeling and Control of Mechanisms and Robots, C. Melchiorri and A. Tornambé, Eds. Singapore: World Scientific, 1996, pp. 81-113.

[2] S. Battilotti, Noninteracting Control with Stability for Nonlinear Systems. Berlin, Germany: Springer Verlag, 1994.

[3] J. Carr, Applications of Centre Manifold Theory. Berlin, Germany: Springer-Verlag, 1981.

[4] I. J. Ha and E. G. Gilbert, "A complete characterization of decoupling control laws for a general class of nonlinear control systems," IEEE Trans. Automat. Contr., vol. AC-31, pp. 823-830, 1986.

[5] H. J. C. Huijberts and A. J. van der Schaft, "Input-output decoupling with stability for Hamiltonian systems," Math. Contr. Signal Syst., pp. 125-138, 1990.

[6] A. Isidori, Nonlinear Control Systems, 3rd ed. Berlin, Germany: Springer-Verlag, 1994.

[7] A. Isidori and J. W. Grizzle, "Fixed modes and nonlinear noninteractive control with stability," IEEE Trans. Automat. Contr., vol. 33, pp. 907-914, 1988.

[8] A. S. Morse and W. M. Wonham, "Decoupling and pole assignment by dynamic compensation," SIAM J. Contr., vol. 8, no. 3, pp. 317-337, 1970.

[9] H. Nijmeijer and W. Respondek, "Decoupling via dynamic compensation for nonlinear control systems," in Proc. IEEE Conf. Decision and Control, 1986.

[10] H. Nijmeijer and A. J. van der Schaft, Nonlinear Dynamical Control Systems. Berlin, Germany: Springer-Verlag, 1990.

[11] K. G. Wagner, "On nonlinear noninteraction with stability," in Proc. 28th Conf. Decision and Control, Tampa, FL, Dec. 1989.

[12] W. M. Wonham, Linear Multivariable Control: A Geometric Approach, 2nd ed. Berlin, Germany: Springer-Verlag, 1979.

[13] W. M. Wonham and A. S. Morse, "Decoupling and pole assignment in linear multivariable systems: A geometric approach," SIAM J. Contr., vol. 8, no. 1, pp. 1-18, 1970.

[14] W. Zhan, A. Isidori, and T. J. Tarn, "A canonical dynamic extension algorithm for noninteraction with stability for affine nonlinear systems," Syst. Contr. Lett., vol. 17, pp. 177-184, 1991.

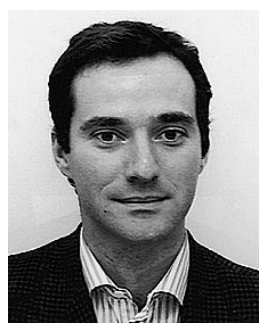

Alessandro Astolfi was born in Rome, Italy, in 1967. He received the degree in electrical engineering from the University of Rome La Sapienza, Italy, in 1991, the Nach-Diploma from ETH-Zurich in 1994, the Ph.D. degree in automatic control from ETH-Zurich in 1995, and the Ph.D. degree in automatic control from University of Rome La Sapienza in 1996.

He was a Lecturer on nonlinear control at ETH-Zurich in 1996 and 1997. Since March 1997, he has been with the Electrical and Electronic Engineering Department and the Centre for Process Systems Engineering, Imperial College, where he is a Lecturer in control engineering. Since 1998 he has been an Associate Professor of control theory at Politecnico of Milan, Italy. His research interests include nonlinear stabilization; control of nonholonomic systems, with emphasis on the problem of discontinuous stabilization; modeling and control of general mechanical systems; and robust control of nonlinear systems, with particular interest in nonlinear $\mathrm{H}$-infinity methods. $\mathrm{He}$ is the author of about 70 papers on international journals and conferences.

$\mathrm{He}$ is an Associate Editor of Systems and Control Letters, European Journal of Control, and International Journal of Control. 


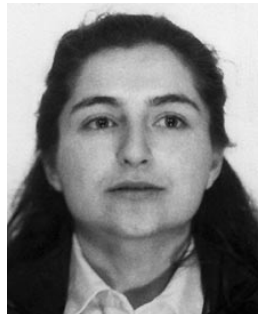

Laura Menini was born in Rome, Italy, in 1970.

She received the laurea degree (summa cum laude)

in mechanical engineering from the University of

Rome Tor Vergata, Italy, in 1993. During 1995 she

was a Visiting Student at the University of New

Mexico at Albuquerque, NM, USA. She received the

Ph.D. degree in computer science and control theory

from University of Rome Tor Vergata in 1997.

Since 1997, she has been a Researcher at the

University of Rome Tor Vergata. Her research interests include nonlinear control; control of Hamiltonian systems; robust control of linear systems, with particular interest in sampled-data systems and periodic systems; multiobjective control; modeling and control of mechanical systems subject to impacts; control of flexible structures; and robots. She is the author of about 30 papers in international journals and conferences. 